\title{
Perspective
}

PERSPECTIVE Actualité en histoire de l'art

1 | 2012

Art et pouvoir

\section{Représentation(s) et autoreprésentation(s) de l'aristocratie romaine}

Representation(s) and self-representation(s) of the Roman aristocracy

\section{Frédéric Hurlet}

\section{(2) OpenEdition \\ 1 Journals}

Édition électronique

URL : http://journals.openedition.org/perspective/605

DOI : $10.4000 /$ perspective.605

ISSN : 2269-7721

Éditeur

Institut national d'histoire de l'art

\section{Édition imprimée}

Date de publication : 30 juin 2012

Pagination : 159-166

ISSN : $1777-7852$

\section{Référence électronique}

Frédéric Hurlet, « Représentation(s) et autoreprésentation(s) de l'aristocratie romaine », Perspective [En ligne], 1 | 2012, mis en ligne le 30 décembre 2013, consulté le 02 octobre 2020. URL : http:// journals.openedition.org/perspective/605; DOI : https://doi.org/10.4000/perspective.605 


\section{Représentation(s) et autoreprésentation(s) de l'aristocratie romaine}

\section{Frédéric Hurlet}

Le statut privilégié de l'Antiquité dans l'histoire et la conscience européennes a longtemps contribué à brouiller les pistes quand il s'est agi d'étudier les relations entre l'art et le pouvoir dans la Rome antique républicaine et impériale. Ce sujet est en soi très complexe et nécessite une méthodologie éprouvée pour aborder les images et interroger ce que le pouvoir d'alors voulait exprimer à travers elles, ou ce que le spectateur antique pouvait ressentir en regardant des inscriptions, des sculptures, des statues ou encore des monuments. La scénarisation de la Rome augustéenne au service du pouvoir fasciste au cours de la première moitié du $\mathrm{XX}^{\mathrm{e}}$ siècle, par exemple lors de la Mostra Augustea della Romanità en 1937-1938 à l'occasion du bimillénaire de la naissance d'Auguste, a renforcé l'idée, commune et erronée, selon laquelle l'art romain était un instrument de propagande au service d'un pouvoir impérial désireux de conditionner l'opinion publique. Actuellement, nous sommes heureusement loin d'une telle simplification. Depuis plusieurs décennies, nous avons appris que le langage visuel, loin d'être imposé par le pouvoir central, était parfois perçu comme une forme de dialogue et de négociation entre le prince, les commanditaires des œuvres et leurs destinataires. Les travaux de Tonio Hölscher et de Paul Zanker ont ouvert la voie et transformé l'histoire de l'art et l'archéologie classique en analysant les images comme des systèmes sémantiques qui renvoient à un contexte et à des réalités politiques, sociales et culturelles ${ }^{1}$. Ce n'est sans doute pas un hasard si cette nouvelle approche est née dans les deux pays, Italie et Allemagne, dont le passé fasciste avait déjà fait ressortir ce que Paul Zanker a appelé « le pouvoir des images $"{ }^{2}$. On citera également les analyses de Pierre Gros sur la mise en scène du pouvoir impérial à Rome et dans les cités de l'Empire ${ }^{3}$. Cependant, ces travaux portant sur le dialogue entre gouvernants et gouvernés ont rarement abordé la figure de l'aristocrate, incarné par le sénateur, qui exerça la fonction de médiateur entre le prince et ses administrés. Le bilan qui suit a pour objet d'étudier cet acteur tel qu'il apparaît à travers les images et en relation avec sa position dans les hauts rangs de l'État. L'accent sera mis sur l'évolution qui fit progressivement de ces dirigeants naturels sous la République des administrateurs de l'Empire à partir d'Auguste.

\section{L'aristocratie romaine dans les recherches actuelles}

L'historiographie a fait de l'aristocratie romaine aussi bien pendant la République qu'au début de l'époque impériale un thème en vogue. L'intérêt pour ce sujet est largement le fait du développement et des succès de la méthode prosopographique pendant une grande partie du $\mathrm{XX}^{\mathrm{e}}$ siècle, à la suite des travaux fondateurs publiés par Matthias Gelzer en 1912 et par Friedrich Münzer en $1920^{4}$. C'est dans ce courant que s'inscrivent les analyses de Ronald Syme, auteur de La Révolution romaine, livre paru en $1939^{5}$, qui fut le meilleur spécialiste de l'aristocratie augustéenne. Les paradigmes interprétatifs se sont toutefois modifiés depuis trois décennies. Concernant la République romaine, les études de Fergus Millar publiées depuis les années 1980 
1. Autel des Lares provenant du Vicus Sandalarius, 2 avant J.-C., Rome. Auguste, au centre, tient un lituus. ont relativisé le poids de l'aristocratie dans le système politique et insisté sur les différentes formes de participation active du peuple à la vie publique, allant même jusqu'à parler de démocratie à Rome ${ }^{6}$. À propos de l'Empire, la figure du prince et les productions artistiques qui lui ont été consacrées ont retenu légitimement l'attention des chercheurs, le point d'orgue de cette évolution étant l'ouvrage de Paul Zanker sur Auguste publié en $1987^{7}$.

Les avancées scientifiques intervenues depuis les années 1980 ne suggèrent toutefois pas qu'il faille sous-estimer la place de l'aristocratie dans le système politique de la République à l'Empire, Auguste se comportant comme un aristocrate et en fonction des valeurs de cette couche sociale. Karl-Joachim Hölkeskamp a ainsi montré que, en dépit de ce qu'a écrit Millar, la « culture politique " était restée foncièrement aristocratique à Rome tout au long de la République ${ }^{8}$. Un des principaux apports d'un colloque organisé à Nantes en 2007 sur le thème de la restauration de la Res publica par Auguste est d'avoir montré que la naissance du Principat ne modifia pas foncièrement cet état de fait car les cultures monarchique et traditionnelle coexistèrent, en tout cas au début de cette période ${ }^{9}$. Auguste prit en effet le pouvoir et fonda un nouveau régime tout en affirmant restaurer la Res publica. Il n'a de ce fait pas aboli les anciennes institutions, mais il s'est surimposé à celles-ci. L'aristocratie romaine a donc continué à être présente dans le processus de prise de décisions, bien qu'étant en retrait d'Auguste et de sa famille.

L'image de l'aristocratie ne disparut pas pour autant de l'espace public, l'enjeu principal étant de l'articuler avec celle du prince. En effet, l'(auto)représentation de l'aristocrate apparaît comme un phénomène d'autant plus central qu'elle s'inscrivait dans un cadre public. C'est la conclusion à laquelle conduisent non seulement l'ouvrage de synthèse de Hölkeskamp sur la République romaine, mais aussi l'analyse des images. Si l'on observe la représentation de la vie politique à travers les sources figurées, il apparaît, comme l'a rappelé Raphaëlle Laignoux, que la vocation de l'aristocrate à gouverner n'a jamais été remise en cause sous la République, même au plus fort de la crise ${ }^{10}$. La supériorité

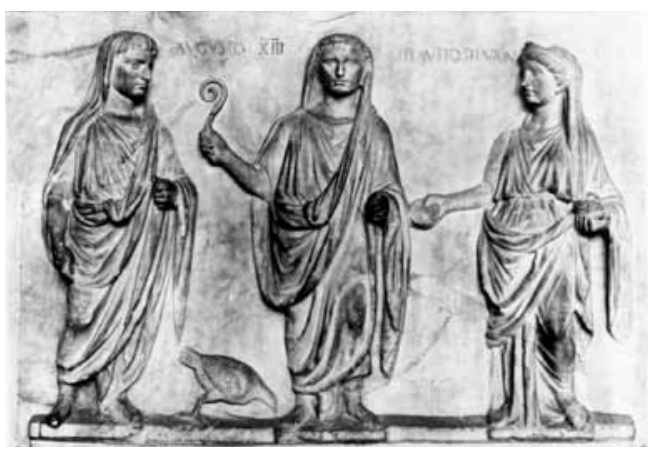

symbolique du magistrat romain ne se discutait pas; la présence sur les monnaies et les reliefs d'attributs du pouvoir tels que les faisceaux, les licteurs ou encore le lituus - bâton recourbé lié à la prise d'auspices et donc à l'exercice de toute forme de pouvoir (fig. l) - l'a d'ailleurs prouvé.

\section{L'aristocratie en représentation : inscriptions, monnaies, architecture}

Un des acquis des recherches historiographiques sur la République romaine de cette dernière décennie est d'avoir révélé dans quelle mesure la vie publique à Rome était marquée par la participation du populus Romanus aux prises de décision, que ce soit pour peser sur ce processus ou pour ratifier des mesures déjà prises par les sénateurs. Cette mise en valeur de la notion de publicité explique la dimension théâtrale de la politique romaine, qui prit à de nombreuses occasions l'allure d'une scène saturée d'images de toutes sortes. Gilles Sauron a ainsi illustré la facilité avec laquelle les chefs de l'aristocratie s'assimilaient aux héros de tragédie et aux dieux, à Énée et à Apollon dans le cas d'Octavien, devenu Auguste ${ }^{11}$.

Parmi les multiples images publiques qu'il faut interpréter comme autant de stratégies de communication au sein de l'aristocratie ou entre celle-ci et le peuple, l'intérêt des historiens s'est porté légitimement sur les documents épigraphiques. Les inscriptions placées sur différents supports et attestées en grand nombre dans la documentation existante donnèrent à voir aux spectateurs une image de l'aristocratie qui resta très répandue. Le Principat d'Auguste est une période charnière qui coïncida avec la naissance d'une culture épigraphique matérialisée par une multiplication du nombre des inscriptions, 


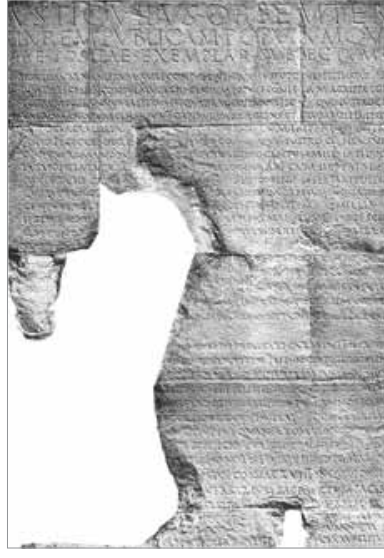

phénomène qui a été analysé en particulier par Geza Alföldy ${ }^{12}$. Étant donné les découvertes régulières dans ce domaine, l'épigraphie est considérée comme un champ capable de renouveler notre connaissance de l'aristocratie romaine. Or, comme Werner Eck l'a souligné, l'inscription, loin d'être uniquement une source écrite, est aussi figurée, c'est-à-dire indissociable du monument auquel le texte épigraphique appartient, même si elle pouvait apparaître comme visuellement secondaire par rapport au contexte architectural, parfois grandiose, qui l'environnait et qu'elle contribuait à éclairer ${ }^{13}$. C'est également ce qu'a souligné Jas Elsner en rappelant à propos des Res Gestae Diui Augusti que ce célèbre document épigraphique, avant d'être un texte, était une inscription monumentale localisée à Rome dans un contexte topographique lié au nouveau régime - le mausolée d'Auguste (fig. 2) ${ }^{14}$.

Les épigraphistes connaissent la pratique qui consiste à énumérer sur le piédestal d'une statue l'ensemble des fonctions exercées par un sénateur - plus connue sous le nom de carrière sénatoriale. Eck a montré que cet usage a connu une évolution remarquable. À l'époque républicaine, il était habituel d'inscrire du vivant du sénateur honoré uniquement la dernière magistrature exercée, qui était la plus élevée du cursus honorum, ou éventuellement une des grandes prêtrises. Sur les dédicaces à Sylla par exemple, ce dernier est qualifié à la fin de sa vie de " dictateur " sans que ses autres magistratures précédentes ne soient citées. Le phénomène de la concurrence aristocratique se révélait pleinement dans cette stratégie discursive car l'âge était rappelé uniquement dans les cas de sénateurs décédés prématurément qui n'avaient pas revêtu de magistratures ou atteint la plus élevée d'entre elles ; la pression sociale était si forte au sein des grandes gentes de l'aristocratie que l'on justifiait ainsi le non-exercice du consulat par le décès, comme l'ont montré Hölkeskamp et $\mathrm{Eck}^{15}$. C'est à partir de l'époque augustéenne que fut développé sur la dédicace l'ensemble du cursus honorum d'un sénateur de son vivant, depuis la première jusqu'à la dernière fonction et dans des ordres déterminés (direct ou inverse), préfigurant la pratique de l'époque impériale. Eck explique cette évolution par deux facteurs, qui se renforcent plus qu'ils ne s'excluent : d'une part la volonté de mettre en avant le patronage du prince, d'autant que ce dernier ne se limitait pas à intervenir dans l'octroi de la dernière magistrature ou fonction ; d'autre part le modèle des inscriptions du Forum d'Auguste, gravées sur les piédestaux des statues des sénateurs gratifiés des ornements triomphaux - principale décoration militaire, abordée plus loin - et qui présentaient la particularité par rapport aux autres inscriptions de décrire toutes les activités publiques d'un sénateur alors en vie ${ }^{16}$.

Il ne faut pas non plus négliger l'étude des sources numismatiques, qui a fait l'objet d'avancées ces deux dernières décennies. Liée dès l'Antiquité à toute forme de pouvoir, la monnaie fut frappée par l'État romain à l'époque républicaine et sous Auguste, aussi bien à Rome qu'hors de la cité dans le cadre des monnayages de campagne. Le premier tome de Roman Provincial Coinage, paru en 1992 sous la direction de Andrew Burnett, de Michel Amandry et de Pere Pau Ripolles, et consacré en partie à la période augustéenne, fut le principal apport sur cette question. Ce catalogue de monnaies met à la disposition des historiens et des historiens de l'art des sources autrefois d'accès difficile ${ }^{17}$. Le monnayage provincial romain désigne les frappes civiques ou provinciales intégrées au système romain, mais émises par des autorités autres que le pouvoir central romain. Dans le courant du $\mathrm{I}^{\mathrm{er}}$ siècle, au moment de la montée en puissance des imperatores (Marius, Sylla et Pompée), des privilèges iconographiques furent accordés aux aristocrates sur les monnaies, quoique de façon discrète et par empiètements successifs. Comme l'a montré Arnaud Suspène, il fallut attendre l'année 44 pour qu'une décision officielle honorât de son vivant un sénateur, Jules César, d'un portrait monétaire sur le droit
2. Fragment des Res Gestae Diui Augusti. 
en vertu d'un sénatus-consulte ${ }^{18}$. À l'époque augustéenne, le portrait du prince et ceux des membres de sa dynastie devinrent très rapidement le trait distinctif de la monnaie romaine, quelle que fût l'origine du monnayage. Toutefois, une exception demeure : des monnaies provinciales provenant d'Asie et d'Afrique représentent plusieurs proconsuls d'Afrique et d'Asie (neuf en tout) sur le droit ou le revers, parfois, sur le droit, en relation avec l'image d'Auguste et de l'un ou l'autre membre de sa famille. Limitées à certaines périodes et aux proches du prince, elles témoignent de la collaboration nécessaire entre le pouvoir impérial et l'aristocratie, ainsi que de la permanence d'une concurrence et d'une hiérarchisation au sein de ce groupe social ${ }^{19}$.

L'aristocratie romaine privilégiait également la maison comme espace de représentation visà-vis des autres Romains, en particulier par sa localisation, par son agencement et par son décor. À Rome, l'habitat n'était jamais neutre ni totalement privé, mais il se chargeait de signification politique et sociale ${ }^{20}$. Sans aller jusqu'à identifier un type standardisé de domus propre à une catégorie sociale déterminée, les travaux récents, de Henner von Hesberg en particulier, ont souligné la dimension politique d'un lieu qui permettait à son propriétaire de renforcer sa visibilité sur la scène publique ${ }^{21}$. La maison était le cadre topographique d'une série de manifestations qui le mettaient en contact avec un nombre plus ou moins élevé de Romains : avec ses clients au cours de la salutatio matinale ou avec ses amis à l'occasion de rencontres plus informelles. Le caractère aristocratique de la société romaine à l'époque tardo-républicaine conduisit les gentes en vue et leurs principaux membres à une compétition qui fit de la maison un critère de différenciation et, de ce fait, un des lieux du pouvoir. On connaît une liste d'aristocrates qui se firent construire des maisons de plus en plus somptueuses, depuis le consul de 95 L. Crassus jusqu'à l'édile de 58 M. Aemilius Scaurus. Un des moyens matériels de cette concurrence fut l'usage du marbre, notamment quand furent intégrés au sein de la résidence des éléments de décoration propres à l'architecture publique, tels que des séries de colonnes. De ce point de vue, l'atrium, lieu public par excellence, était la pièce centrale de la domus romaine.
La synthèse présentée par Pierre Gros en donne une analyse enrichissante ${ }^{22}$. La naissance du Principat fut marquée par la prise de possession de la colline du Palatin par le nouveau régime, processus qui a été décrit par Pierre Gros pour ses premières étapes augustéennes et qui déboucha à la fin de l'époque julio-claudienne sur la construction d'un véritable complexe palatial ${ }^{23}$. Cependant, avec l'installation de ce " quartier impérial ", les domus de l'aristocratie augustéenne ne perdirent pas leur principale fonction politique, qui était de mettre en avant le statut du propriétaire. Les parties de ces maisons consacrées à l'otium, le loisir, gagnèrent progressivement en importance et en signification, phénomène que Von Hesberg qualifie $\mathrm{d}^{\prime}$ " introversion » 24 .

\section{L'art et l'image comme représentation des rituels sociaux}

Martin Jehne a fait des ouvrages de Claude Nicolet sur le métier de citoyen et de Paul Veyne sur le pain et le cirque, publiés tous deux en 1976, un jalon historiographique qui a consisté à accorder une plus grande attention au mode de communication politique entre les élites et le peuple ${ }^{25}$. À Rome, le dialogue passait par l'image, en particulier par la représentation figurée de cérémonies publiques au centre desquelles se trouvait la figure de l'aristocrate. Le triomphe était l'une d'entre elles, sans doute la plus importante et la plus étudiée cette dernière décennie, car le défilé du vainqueur, de ses troupes, du butin et des prisonniers était une mise en scène destinée à souligner devant le public la majesté du peuple Romain et l'excellence du général ${ }^{26}$. L'image la plus répandue du triomphe était l'arc honorifique érigé sur le parcours du défilé (on songe au fornix Scipionis sur le Capitole et au fornix Fabianus sur le Forum). Ce monument peut être défini comme un "lieu de mémoire", selon l'analyse de Hölkeskamp, dans le sens où il inscrit dans le marbre le souvenir d'un des rituels les plus représentatifs du fonctionnement de l'aristocratie ${ }^{27}$. Il pouvait être décoré de reliefs qui rappelaient des scènes de cette cérémonie, comme on le voit sur l'arc de Titus placé entre le Colisée et le Capitole et commémorant le double triomphe de Vespasien et de Titus en 71 après J.-C. Pour la fin de la République, la figure 


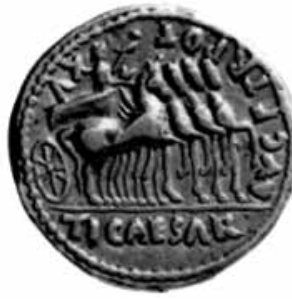

la plus spectaculaire du triomphateur attestée par la numismatique est celle de l'imperator - Marius, Sylla ou Pompée - représenté sur le char triomphal, le quadrige, en compagnie de leurs fils pour Marius et Pompée, de manière à insister sur l'aspect dynastique de tout pouvoir à Rome. Les silhouettes restent toutefois discrètes, et elles ne sont d'ailleurs pas identifiées par une légende monétaire ; elles renvoient donc à une (auto)représentation prudente. L'avènement du Principat modifia la situation en réservant au prince et aux membres de sa dynastie le monopole du triomphe à partir de 19 avant J.-C. et en les assimilant à des vainqueurs par excellence. Une statue d'Auguste sur son quadrige fut érigée au centre de son forum en référence à son triple triomphe d'août 29 ; quant à Tibère, successeur désigné à partir de 4 après J.-C., avec son adoption et son association aux pouvoirs impériaux, il figure comme triomphateur sur le revers d'un type monétaire daté de l'extrême fin du Principat d'Auguste (13-14 après J.-C.) : il y apparaît lauré, debout à droite dans un quadrige, tenant un laurier et un sceptre surmonté d'un aigle (fig. 3) ${ }^{28}$. Il faut y voir une référence à son second triomphe, celui sur l'Illyrie, célébré le 23 octobre 12 après J.-C. La posture est très proche de celle que l'on peut observer sur une des coupes de Boscoreale, qui commémore l'un des triomphes de Tibère, celui de 7 avant J.-C. ou de 12 après J.-C., et qui a été étudiée en profondeur par Ann Kuttner ${ }^{29}$.

Des mécanismes de compensation furent rapidement mis en place pour l'aristocratie augustéenne avec l'apparition dans le courant des années 10 avant J.-C. des ornements triomphaux, analysés par $\mathrm{Eck}^{30}$. Ce nouveau type de décoration militaire, hiérarchiquement inférieur au triomphe ou à l'ovation (triomphe à cheval), était attribué aux sénateurs ayant remporté une victoire significative. Il trouva une traduction visuelle publique à partir de 2 avant J.-C. avec une disposition de la loi sacrée du temple de Mars Ultor prescrivant qu'il fallait ériger dans l'enceinte du forum d'Auguste des statues en bronze des triomphateurs et de ceux auxquels les ornements triomphaux étaient décernés (Dion, LV, 10, 3).

Les funérailles du noble romain, désignées sous la formule de pompa funebris, constituaient une autre cérémonie attestée sur les sources figurées. Décrites par Polybe comme un des traits distinctifs de l'aristocratie romaine (Polybe, VI, 53-54) ${ }^{31}$, elles se déroulaient selon un rituel dont une étape bien connue était le défilé des portraits en cire des ancêtres de la famille de la personne décédée (imagines). Ceux-ci prenaient sans doute la forme de mannequins transportés après avoir été habillés en fonction des honores accordés à chacun des ancêtres ${ }^{32}$. Selon Harriet Flower, cette mise en scène de la mort et du mort était un indice et un symbole visuel du rang tenu par la famille noble, aussi bien sous la République que tout au long du Principat augustéen, puisque seules étaient présentes les imagines des ancêtres qui avaient exercé des magistratures et dont la mémoire n'avait pas été condamnée ${ }^{33}$. Le caractère concurrentiel de la culture politique romaine explique que cette partie si caractéristique du rituel ait été représentée dans l'imagerie, mais finalement peu souvent. Outre un relief provenant d'Amiternum, le document iconographique le plus connu reste la statue "Barberini " (fig. 4). Pendant longtemps, on vit dans cette ouvre un noble romain inconnu, identifié à tort à Lucius Iunius Brutus, revêtu de la toge et portant dans chaque main un portrait des ancêtres à des niveaux différents, peut-être le père pour le plus haut et le grand-père pour le plus bas. Cette interprétation traditionnelle a été toutefois récemment remise en cause par Petra Cain, qui a montré qu'une des têtes portées était celle d'une femme ${ }^{34}$. Il est difficile de donner une datation précise en dehors de la période tardo-républicaine ou augustéenne, car l'ensemble a été
3. Aureus représentant le triomphe de Tibère en 12 après J.-C., atelier impérial de Lyon, 13-14 après J.-C.
4. Statue "Barberini", fin $I^{\text {er }}$ siècle avant J.-C., Rome, Musei Capitolini. 
5. Ara Pacis, 13-9 avant J.-C., Rome, Museo dell'Ara Pacis, détail de la frise méridionale sur laquelle on aperçoit à gauche Auguste, à I'extrême droite Agrippa et au milieu une série de personnages dont les flamines, reconnaissables par le port de l'apex (bonnet à pointe). remanié et a fait l'objet d'additions. La tête y a été fixée seulement à l'époque moderne et est communément datée du $\mathrm{I}^{\mathrm{er}}$ siècle avant J.-C., tandis que les plis de la toge et le portrait de gauche placent le reste de la sculpture à l'époque augustéenne $^{35}$.

À la suite du défilé et de l'éloge prononcé publiquement par le fils du défunt, le corps était incinéré et les cendres déposées dans un mausolée, ultime étape d'autant plus marquante que le monument funéraire était imposant. Les aristocrates de la fin de la République prirent l'habitude d'en faire construire à la sortie de Rome, comme celui qu'on appelle le tombeau de Caecilia Metella, situé au début de la Via Appia. À l'époque augustéenne, l'aristocratie continua à faire ériger des monuments funéraires impressionants, comme en témoigne le mausolée des Plautii bâti à Tibur sous Auguste. Mais ce dernier surpassa tout ce qui s'était fait jusqu'alors en faisant construire son mausolée à Rome, sur le champ de Mars, dès la fin des années 30 avant J.-C., monument qui a été étudié par Von Hesberg ${ }^{36}$. Se mit ainsi en place le processus qui réserva très vite à la famille impériale les espaces publics de Rome et qui conduisit les grandes familles aristocratiques à faire édifier des statues ou des monuments en leur honneur dans les villes d'Italie et des provinces. Eck a largement décrit ces modalités spatiales de la concurrence sous le Haut-Empire ${ }^{37}$. Selon lui, l'aristocratie sénatoriale pouvait poursuivre librement ailleurs qu'à Rome ses rêves de prestige personnel et de memoria de longue durée, ainsi que sa tendance naturelle à la compétition.

L'imitation par les cités provinciales de monuments romains directement liés aux décès des membres de la famille impériale et des rites qui y étaient pratiqués est un phénomène qui a été mis en évidence ces dernières années ${ }^{38}$. C'est ainsi que des arcs honorifiques furent construits dans les provinces en mémoire de Germanicus, sur le modèle de celui édifié à Rome près du Circus Flaminius, et que des temples furent érigés à des fins funéraires, comme ce fut le cas de la Maison carrée de Nîmes, élevée ou reconvertie en l'honneur des princes de la jeunesse à la suite de leurs décès au tout début de notre ère ${ }^{39}$. Il faut ajouter l'autel devant lequel étaient célébrés chaque année les sacrifices publics destinés à honorer les mânes des princes défunts le

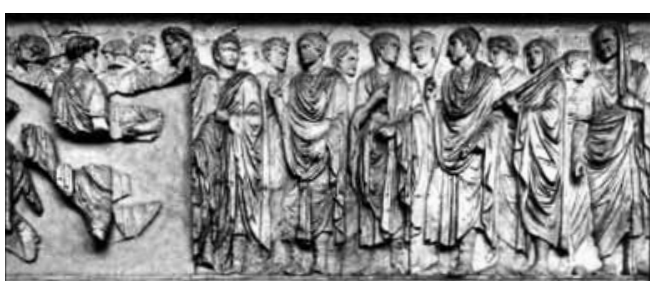

jour anniversaire de leurs morts, et qui était situé à Rome devant le mausolée d'Auguste (le même phénomène est attesté aussi à Pise).

La meilleure représentation en images de l'écart qui se créa sous Auguste entre les membres de la dynastie et les autres aristocrates romains dans la mise en scène de cérémonies publiques se trouve sur les frises centrales de l'Ara Pacis, reproduisant une procession liée à une supplicatio (fig. 5). De nombreux sénateurs y étaient représentés non pas sous des traits personnalisés, mais comme des prêtres appartenant à un des grands collèges religieux de Rome, le flaminat. Le contraste avec les figures des membres de la famille impériale est frappant, car ces derniers, nombreux à apparaître sur les reliefs, sont davantage individualisés ${ }^{40}$.

Il a déjà été écrit que l'art romain était un art politique et social. Il servait à ce titre à représenter des dignitaires, des monuments publics et des rituels, et contribuait à forger dans la durée une identité collective. Le sénateur y apparaît comme une figure centrale tout au long de la République en vertu du " capital symbolique " qu'il détenait ${ }^{41}$ et qui donnait à l'aristocratie une vocation à gouverner. La naissance du Principat modifia la situation en plaçant à la tête de la Res publica un prince, qui était un aristocrate de haut rang en raison de son adoption par Jules César. Ce changement de régime eut pour conséquence d'imposer l'image d'Auguste et de sa dynastie dans l'espace public, que ce soit sur les monnaies, les inscriptions, les statues ou les reliefs. Toutefois une telle monopolisation fut loin d'être un processus linéaire car la représentation de l'aristocratie romaine fut progressivement supplantée par celle du pouvoir impérial, sans jamais disparaître totalement. La raison de cette moindre visibilité est d'ordre politique. La lutte féroce pour le pouvoir si caractéristique du fonctionnement de l'aristocratie de la fin de la République fit place à partir d'Auguste 
à une compétition dont l'intensité resta forte à l'échelle d'un cursus honorum sénatorial élargi mais qui fut désormais arbitrée par le prince.

1. Adolf H. Borbein, Tonio Hölscher, Paul Zanker éd., Klassische Archäologie: Eine Einführung, Berlin, 2000.

2. Voir à ce sujet le compte rendu de l'ouvrage de Paul Zanker, Augustus und die Macht der Bilder, par Andrew Wallace-Hadrill : "Rome's Cultural Revolution", dans The Journal of Roman Studies, 79, 1989, p. 157-164.

3. Pierre Gros, L'Architecture romaine, I, Les monuments publics, Paris, 1996.

4. Matthias Gelzer, Die Nobilität der römischen Republik, Leipzig, 1912 ; Friedrich Münzer, Römische Adelsparteien und Adelsfamilien, Stuttgart, 1920.

5. Ronald Syme, La Révolution romaine, Paris, 1967 [éd. orig. : The Roman Revolution, Oxford, 1939].

6. On consultera à ce sujet le recueil suivant : Fergus Millar, Rome, the Greek World, and the East, I, The Roman Republic and the Augustan Revolution, Hannah M. Cotton, Guy M. Rogers éd., Chapell Hill/Londres, 2002, en particulier p. 85-182.

7. Paul Zanker, Augustus und die Macht der Bilder, Munich, 1987.

8. Karl-Joachim Hölkeskamp, Reconstruire une République : la "culture politique " de la Rome antique et la recherche des dernières décennies, Nantes, 2008 [éd. orig. : Rekonstruktionen einer Republik: die politische Kultur des antiken Rom und die Forschung der letztzen Jahrzehnte, Munich, 2004].

9. Frédéric Hurlet, Bernard Mineo éd., Le Principat d'Auguste réalités et représentations du pouvoir, autour de la Res publica restituta, Rennes, 2009.

10. Raphaëlle Laignoux, La Construction du pouvoir personnel durant les années 44-29: processus de légitimation, thèse, université Paris 1 Panthéon-Sorbonne, 2010.

11. Gilles Sauron, « Du triumvirat au Principat : la construction du mythe augustéen ", dans Hurlet, Mineo, 2009, cité n. 9, p. 187-208.

12. Géza Alföldy, " Augustus und die Inschriften: Tradition und Innovation. Die Geburt der imperialen Epigraphik ", dans Gymnasium, 98, 1991, p. 289-324.

13. Werner Eck, Monument und Inschrift: Gesammelte Aufsätze zur senatorischen Repräsentation in der Kaiserzeit, Walter Ameling, Johannes Heinrichs éd., Berlin, 2010, p. 7-10.

14. Sur le statut textuel et visuel de l'inscription, voir Jas Elsner, "Inventing Imperium: Texts and the Propaganda of Monuments in Augustan Rome ", dans Jas Elsner éd., Art and Text in Roman Culture, Cambridge, 1996, p. 32-53.

15. Hölkeskamp, 2008, cité n. 8, p. 108-111 ; Eck, 2010 , cité n. 13, p. 255-256.
16. Werner Eck, " Der Senator und die Öffentlichkeit - oder: wie beeindruckt man das Publikum? ", dans Werner Eck, Matthäus Heil éd., Senatores populi Romani: Realität und Mediale Präsentation einer Führungsschicht, Stuttgart, 2005, p. 8-9.

17. Andrew Burnett, Michel Amandry, Pere Pau Ripolles éd., Roman Provincial Coinage, I, Londres/Paris, 1992.

18. Arnaud Suspène, "Les effigies monétaires romaines et l'apparition du portrait de César : problèmes légaux et politiques ", dans Revue des études anciennes, 110, 2008, p. 461-481.

19. Voir Frédéric Hurlet, Arnaud Suspène, "Le proconsul et le prince. À propos des portraits monétaires des proconsuls d'Afrique et d'Asie sous le Principat d'Auguste ", dans La société romaine et ses élites, hommages à Élizabeth Deniaux Paris, à paraître.

20. On consultera l'ouvrage de Annapaola Zaccaria Ruggiu, Spazio privato e spazio pubblico nella città romana, Rome, 1995. Voir aussi plus récemment Renaud Robert, "Priuata modo et domestiqua nos délectant. Espace domestique, espace utopique? ", dans Chiara Carsana, Maria Teresa Schettino éd., Utopia e utopie nel pensiero storico antico, Rome, 2008, p. 111 132, et Shelley Hales, The Roman House and Social Identity, Cambridge, 2008.

21. Henner von Hesberg, "Die Häuser der Senatoren in Rom: Gesellschaftliche und politische Funktion », dans Eck, Heil, 2005, cité n. 16, p. 19-52.

22. Pierre Gros, L'architecture romaine, II, Maisons, palais, villas et tombeaux, Paris, 2001, p. 72-77.

23. Pierre Gros, "Les limites d'un compromis historique : de la domus vitruvienne à la maison augustéenne du Palatin ", dans Hurlet, Mineo, 2009, cité n. 9, p. 169-185.

24. Henner von Hesberg, "Die Häuser der Senatoren in Rom: Gesellschaftliche und politische Funktion », dans Eck, Heil, 2005, cité n. 16, p. 42-45.

25. Martin Jehne parle d'un " communicative turn " dans "Methods, Models, and Historiography ", dans Nathan Rosenstein, Robert Morstein-Marx éd., A Companion to the Roman Republic, Oxford, 2006, p. 12-13.

26. Sur le triomphe, voir Mary Beard, The Roman Triumph Harvard, 2007, et Ida Ostenberg, Staging the World Spoils Captives, and Representations in the Roman Triumphal Procession, Oxford, 2009.

27. Karl-Joachim Hölkeskamp, « Der Triumph - 'Erinnere Dich, daß Du ein Mensch bist' ", dans Elke Stein-Hölkeskamp, Karl-Joachim Hölkeskamp éd., Erinnerungsorte der Antike: Die römische Welt, Munich, 2006, p. 258-276.

28. Carol Humphrey Vivian Sutherland, Roman Imperial Coinage, $\mathrm{I}^{2}$, Londres, 1984, p. 221-224.

29. Ann Kuttner, Dynasty and Empire in the Age of Augustus: The Case of the Boscoreale Cups, Berkeley/Los Angeles/ Oxford, 1995.

30. Eck, 2010, cité n. 13, p. 241-249.

31. On consultera l'ouvrage de Christophe Badel, La Noblesse de l'empire romain : les masques et la vertu, Paris, 2005. 
32. L'idée selon laquelle des masques étaient portés par les figurants au cours des funérailles a été contestée : Heinrich Drerup, "Totenmaske und Ahnenbilder bei des Römern ", dans Römische Mitteilungen, 87, 1980, p. 81-129, et Peter Blome, "Die imagines maiorum: Ein Problemfall römischer und neuzeitlicher Ästhetik ", dans Gottfried Boehm éd., Homo Pictor, Munich/Leipzig, 2001, p. 305-322.

33. Harriet Flower, «Der Leichenzug - die Ahnen kommen wieder ", dans Stein-Hölkeskamp, Hölkeskamp, 2006, p. 321-337, cité n. 27.

34. Voir Klaus Fittschen, Paul Zanker, Petra Cain, Katalog der römischen Porträts in den Capitolinischen Museen und den anderen kommunalen Sammlungen der Stadt Rom, II, 2010, p. 48-51. Pour la statue, voir $\mathrm{n}^{\circ} 38$, pl. 40-41, suppl. 30 b et $31 \mathrm{c}-\mathrm{d}$; Pour la tête, $n^{\circ} 28$, pl. 30-40.

35. Voir les remarques de Harriet Flower, Ancestor Masks and Aristocratic Power in Roman Culture, Oxford, 1996, p. 5-6.

36. Henner von Hesberg, "Das Mausoleum des Augustus der Vater des Vaterlandes und sein Grabmal ", dans SteinHölkeskamp, Hölkeskamp, 2006, cité n. 27, p. 340-361.

37. Werner Eck, "Emperor and Senatorial Aristocracy in Competition for Public Space ", dans Björn C. Ewald, Carlos F. Norena éd., The Emperor and Rome: Space, Representation, Ritual, Cambridge, 2010, p. 89-110.

38. Voir à ce sujet Frédéric Hurlet, "Les modalités de la diffusion et de la réception de l'image et de l'idéologie impériale sous le Haut-Empire en Occident ", dans Milagros Navarro Caballero, Jean-Michel Roddaz éd., La Transmission de l'idéologie impériale dans l'Occident romain, Bordeaux, 2006, p. 49-68.

39. Voir à ce sujet Michel Christol, Dominique Darde éd., L'Expression du pouvoir au début de l'Empire : autour de la Maison Carrée à Nîmes, Paris, 2009.

40. Dietrich Boschung, "Ordo senatorius: Gliederung und Rang des Senats als thema der römischen Kunst ", dans Eck, Heil, 2005, cité n. 16, p. 97-110.

41. Sur la notion de "capital symbolique", voir les ouvrages de Pierre Bourdieu : Raisons pratiques, Paris, 1994, p. 116-123 et p. 187-189; Esquisse d'une théorie de la pratique, Paris, 2000, p. 348-376; Langage et pouvoir symbolique, Paris, 2001, p. 295.

Frédéric Hurlet, Université de Nantes frederic.hurlet@wanadoo.fr

\section{Mots-clés}

aristocratie, autoreprésentation, Principat augustéen, République romaine, rituel 